\section{PARTICLE TRAJECTORIES FOR COMPTON SCATTERING IN ONE SPACE DIMENSION}

ADRIANA SCANTEIANU, XIANGYUE WANG

\section{* ABSTRACT}

Using a relativistic extension of Bohmian Mechanics known as Multi-Time Wave Function formulation, we examine a two-body, one-dimensional system consisting of one photon and one electron that interact only upon contact. We investigate the effects that various parameters in this theory including momentum of the incoming photon and mass of the electron have on the dynamics of the two interacting bodies with the goal of understanding conservation of momentum and energy in the system. We show that the core principles of Compton scattering remain when we use this alternative formulation of quantum mechanics. Although a complete relativistic theory of Bohmian mechanics has yet to be developed, our work aims to make the ideas in this theory more accessible to a wider audience.

\section{$1 \quad$ INTRODUCTION}

Mathematical physics aims to provide logically consistent and mathematically rigorous explanations for why nature behaves the way it does. Currently, at the fundamental level, there are two experimentally verified and mathematically rigorous theories that seem to be in conflict: namely, quantum mechanics and general relativity. While quantum field theory is, for all practical purposes, highly successful at reconciling quantum mechanical principles with Einstein's theory of light (special relativity), it is incompatible with Einstein's theory of gravitation (general relativity). It is also mathematically non-rigorous due to its inherent infinities ${ }^{[14]}$. Such incompatibilities and lack of rigor have led mathematical physicists to continue exploring other theoretical frameworks. More than half a century ago, Louis de Broglie and David Bohm formulated a non-relativistic theory where elementary particles exist regardless of observations $^{[2,4,10]}$. De Broglie-Bohm theory, otherwise known as Bohmian mechanics, is a formulation of quantum mechanics in which subatomic particles in a system have definite positions, guided by the wave function of the system ${ }^{[10]}$. In one of the relativistic extensions of this theory, there is a single multi-time wave function originally proposed by Dirac, defined on the configuration space of the system that guides the motion of all the particles ${ }^{[5]}$. Some researchers believe the photon wave function was never explored due to historical reasons: Dirac completed his quantization of the electromagnetic field prior to discovering his relativistic electron wave equation, and the world of research acted on the first discovery more than the second ${ }^{[1,5,6]}$. Goldstein and others have developed non-relativistic Bohmian mechanics in great mathematical detai ${ }^{[9,10]}$. The remaining challenge is developing a fully relativistic Bohmian mechanical system with a fixed number of particles. Some have claimed that this task is downright impossible ${ }^{[15]}$. Nevertheless, Kiessling and Tahvildar-Zadeh successfully developed a relativistic Bohmian theory for a single photon and, together with Lienert, they have developed an interacting electron-photon theory in one space dimension ${ }^{[11,12]}$. In this paper, we explore whether their theory is applicable to the study of Compton scattering.

Arthur Compton discovered, ninety-six years ago, that when $\mathrm{X}$-rays interact with a graphite plate, the scattered rays have lower frequencies than the incident ones and the difference in the energy of the "X-ray quanta," now called photons, turns into kinetic energy of the electrons on the surface of the material ${ }^{[3]}$. With this experiment, Compton provided evidence for Einstein's 1905 theory, which stated that 
light behaves as particles. We now know this phenomenon as Compton scattering. Quantum field theory provides us with the following narrative about what happens during the interaction: just before the photon smashes into the electron, it abruptly vanishes into nothing. At the same moment, a new photon with a different frequency emerges next to the electron out of thin air, and the two depart as if they have just collided. We would like to explore a different narrative of a photon-electron collision based on Bohmian principles, as provided in the paper by Kiessling et al ${ }^{[11]}$.

The novelty of our work lies in the actualization of previous theory via our modeling of Compton scattering using MATLAB software ${ }^{[11,13]}$. Furthermore, we are the first to use this novel theoretical framework to analyze the effects of changing the momentum of the incoming photon on the trajectories of the scattered photon and electron. We are also the first to investigate the conservation of momentum and applicability of Compton's scattering formula in this system. The framework we use is compatible with many experimentally verified theories in physics, thus we hope that our work will bring us one step closer to a mathematically rigorous understanding of the world around us on the subatomic leve[ ${ }^{[11]}$.

\section{Methodology}

In this paper, we treat both the electron and the photon as relativistic quantum objects. First, we model the trajectories of a single photon in one space dimension using the photon wave function proposed by Kiessling and Tahvildar-Zadeh [12]. Next, we do the same for a single electron using Dirac's relativistic wave equation for the electron ${ }^{[6]}$. We then examine the two-body quantum-mechanical system of a photon and an electron, first assuming that the two do not interact, and then implementing the photon-electron interaction through a boundary condition, as in the paper by Kiessling et al[11]. Afterwards, we review the method Compton used to compute the formula for the scattering angle in his experiment. We then compare Compton's predicted value for momentum gain of the electron with our computed values for this quantity based on our numerical solutions of the guiding equations for the photon and the electron. We then remark on the obstacles that one needs to overcome if one is to rigorously derive such a formula from our Bohmian perspective. Note that our unit of time is the attosecond (1 attosecond $=10^{-18}$ seconds) and our unit of length is 2.997924 angstroms ( 1 angstrom $=10^{-10}$ meters). This allows for the speed of light in a vacuum to be 1 unit of length over time.

\section{i. Single Photon Theory}

The wave function of a photon is a rank-2 bispinor field defined on the configuration spacetime of the photon ${ }^{[12]}$. In one space dimension this means:

$$
\Psi_{p h}(t, s)=\left(\begin{array}{cc}
0 & \chi_{-}(t, s) \\
\chi_{+}(t, s) & 0,
\end{array}\right)
$$

where $t \in \mathbb{R}$ is a time coordinate and $s \in \mathbb{R}$ is a space coordinate. Since a photon is a relativistic quantum particle, its wave function must satisfy a relativistic partial differential equation, which was discovered by Kiessling \& Tahvildar-Zadeh ${ }^{[12]}$. The photon wave equation is a Dirac-type equation, and in one-dimension it reads:

$$
-i \hbar \gamma^{\mu} \frac{\partial \Psi_{p h}}{\partial x^{\mu}}=0,
$$

Where $\hbar$ is the reduced Planck's constant, $x^{0}=c t$, $x^{1}=s, \gamma^{0}=\left(\begin{array}{ll}0 & 1 \\ 1 & 0\end{array}\right)$, and $\gamma^{1}=\left(\begin{array}{cc}0 & -1 \\ 1 & 0\end{array}\right)$; and repeated indices are summed over the range $\mu=0,1$. Here, $c$ is the speed of light in a vacuum. We will be working in units where $c=1$. The photon wave equation can be solved given an initial wave function $\Psi_{p h}(0, s)=\Psi_{p h}^{0}(s)$. Typical initial data corresponding to a photon localized in both position and momentum space (subject to Uncertainty Principle) can be found in FIGURE 1.

In our case, the typical initial data are Gaussian distributions in photon position space. Since the Fourier transform of a Gaussian is a Gaussian, these also have a Gaussian distribution in momentum space. Therefore, there are two parameters that we can set: the mean $k_{0} \in \mathbb{R}$ of the momentum distribution, and the momentum standard deviation $\alpha_{p h}$. Recall that according to the Uncertainty Principle, 

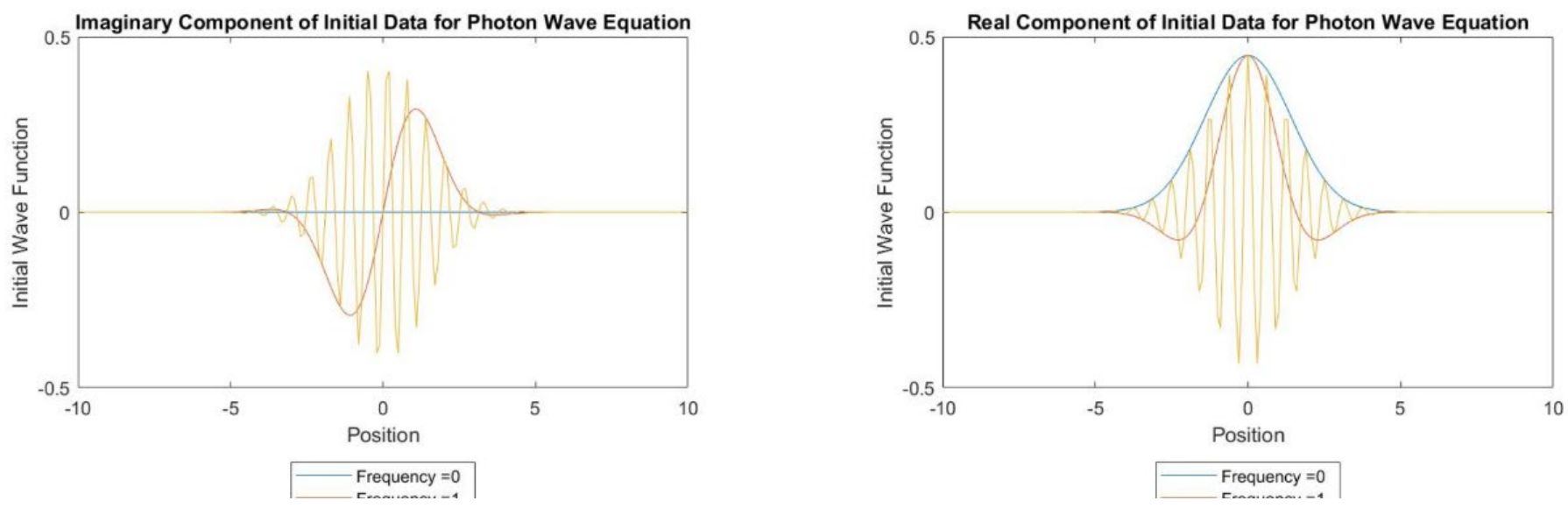

FIGURE 1: Initial data for the photon wave equation is split into real and imaginary components. We assume typical initial data: the photon starts off as a Gaussian wave packet both in position and in momentum-space. Since the unit of frequency is the reciprocal of time, our unit of frequency is $10^{18} \mathrm{~Hz}$.

$\alpha_{p h} \sigma_{p h} \geq \frac{h}{2}$, where $\sigma_{p h}$ is the standard deviation in photon position space, and that this inequality is saturated by the Gaussian distribution. Furthermore, we recall the Einstein-de Broglie energy and momentum relations $E=h v, p=h k$, and the photon dispersion relation $E=|p|$ (recall that $c=1$.) These imply that we may identify $\left|k_{0}\right|$ with the mean of the initial frequency distribution of the photons.

The probability current of detecting a photon at a point in one space and one time dimension is $^{[12]}$ :

$$
j_{p h}^{\mu}(t, s)=\frac{1}{4} \operatorname{Tr}\left(\overline{\Psi_{p h}} \gamma^{\mu} \Psi_{p h} \gamma(X)\right)
$$

where $X=\left(X^{0}, X^{1}\right)$ is a constant vector field computed from $\Psi_{p h}^{0}, \gamma(X):=\gamma_{0} X^{0}+\gamma_{1} X^{1}, \bar{\Psi}:=\gamma^{0} \Psi^{\dagger} \gamma^{0}$, and $\operatorname{Tr}$ denotes matrix trace. The current is conserved: $\partial_{\mu} j_{p h}^{\mu}=0$, future directed $\left(j^{0} \geq 0\right)$, and timelike $\left(j^{0} \geq\left|j^{1}\right|\right)$.

Thus, the probability density of detecting the photon at event $(t, s)$ is $\rho(t, s)=j_{p h}^{0}(t, s)$.

The motion of the photon is guided by its wave function through the following system of guiding equations ${ }^{[11]}$ :

$$
\left\{\begin{array}{l}
\frac{d q}{d t}=v_{p h}(t, q(t))=\frac{c j^{1}(t, q(t))}{j^{0}(t, q(t))} \\
q(0)=q_{0}
\end{array}\right.
$$

Here, $q(t)$ is the position of the photon at time $t ; q_{0}$ is the initial position of the photon. All we know about the initial position is that it is randomly distributed according to the initial probability density $\rho(0, s)$. By solving the system of guiding equations, we obtain the trajectory of a single photon over time.

\section{ii. Single Electron Theory}

According to Paul Dirac, the wave function of a single electron is a spin- $-\frac{1}{2}$ field defined on the configuration space of the electron ${ }^{[6]}$. In one space dimension this means:

$$
\Psi_{e l}(t, s)=\left(\begin{array}{r}
-(t, s) \\
\Psi_{+}(t, s)
\end{array}\right) .
$$

Like in the case of a single photon, the wave function of a single electron also satisfies a relativistic equation. In particular, it satisfies the massive Dirac equation:

$$
-i \hbar \gamma^{\mu} \partial_{\mu} \Psi_{e l}+m_{e l} c \Psi_{e l}=0,
$$

where $m_{e l}$ is the mass of an electron.

The probability current of an electron is:

$$
j_{e l}^{\mu}(t, s)=\overline{\Psi_{e l}} \gamma^{\mu} \Psi_{e l},
$$

where $\bar{\Psi}:=\Psi^{\dagger} \gamma^{0}$ is the Dirac adjoint for rank-one 
bispinors. Furthermore, as in previous research, we define the guiding velocity field of an electron in the same way as that of a photon ${ }^{[11]}$ :

$$
v_{e l}(t, s):=c \frac{j^{1}(t, s)}{j^{0}(t, s)} .
$$

Similarly to the photon case, the guiding equation for the electron is ${ }^{[1]]}$ :

$$
\left\{\begin{array}{l}
\frac{d q}{d t}=v_{e l}(t, q(t))=\frac{c j^{1}(t, q(t))}{j^{0}(t, q(t))} \\
q(0)=q_{0}
\end{array}\right.
$$

The equation satisfied by the electron wave function $\Psi_{e l}$ has a mass term: $\omega=m_{e l} c / \hbar$. In addition, the Gaussian family of initial distributions has a parameter we can change, namely the standard deviation of the probability distribution of electron's initial position: $\sigma$. We will examine the effect of these two parameters on the joint dynamics.

\section{iii. The Two-Body System}

Now, we examine the case in which a photon and an electron are both present, but do not interact. In this case, we have one wave function that guides the motion of each particle through its respective system of guiding equations. The wave function, $\psi$, is a function of four variables, namely the time and position of each particle. To get a wave function that describes both a photon and an electron in a non-interacting system, we take the tensor product $(\otimes)$ of the electron and the photon wave functions, giving us a four component object $\psi=$ $\left(\psi_{--}, \psi_{-+}, \psi_{+-}, \psi_{++}\right)$. The guiding equations for photon and electron are derived using the Hypersurface Bohm-Dirac (HBD) Theory, which allows us to describe the motion of the photon and electron in a common time coordinate ${ }^{[8]}$. The tensored wave function satisfies a relativistic wave equation obtained by the tensor product of the photon and electron wave equations:

$$
\left\{\begin{array}{l}
-i \hbar \gamma^{\mu} \partial_{x_{p h}^{\mu}} \psi=0 \\
-i \hbar \gamma^{\mu} \partial_{x_{e l}^{\mu}} \psi+m_{e l} c \psi=0 \\
\psi\left(0, s_{p h}, 0, s_{e l}\right)=\psi^{0}\left(s_{p h}, s_{e l}\right)
\end{array}\right.
$$

The joint probability current is the following:

$$
\left(\begin{array}{c}
j^{00}=\left|\psi_{--}\right|^{2}+\left|\psi_{-+}\right|^{2}+\left|\psi_{+-}\right|^{2}+\left|\psi_{++}\right|^{2} \\
j^{10}=\left|\psi_{--}\right|^{2}+\left|\psi_{-+}\right|^{2}-\left|\psi_{+-}\right|^{2}-\left|\psi_{++}\right|^{2} \\
j^{01}=\left|\psi_{--}\right|^{2}-\left|\psi_{-+}\right|^{2}+\left|\psi_{+-}\right|^{2}-\left|\psi_{++}\right|^{2} \\
j^{11}=\left|\psi_{--}\right|^{2}-\left|\psi_{-+}\right|^{2}-\left|\psi_{+-}\right|^{2}+\left|\psi_{++}\right|^{2}
\end{array}\right)
$$

Then, the guiding equation for the photon is given by:

$\left\{\begin{array}{l}\frac{d q_{p h}}{d t}=v_{p h}\left(t, q_{p h}(t), t, q_{e l}(t)\right):=\frac{c j^{10}\left(t, q_{p h}(t), t, q_{e l}(t)\right)}{j^{00}\left(t, q_{p h}(t), t, q_{e l}(t)\right)} \\ q_{p h}(0)=q_{p h}^{0}\end{array}\right.$

while the guiding equation for the electron is:

$\left\{\begin{array}{l}\frac{d q_{e l}}{d t}=v_{e l}\left(t, q_{p h}(t), t, q_{e l}(t)\right):=\frac{c j^{01}\left(t, q_{p h}(t), t, q_{e l}(t)\right)}{j^{00}\left(t, q_{p h}(t), t, q_{e l}(t)\right)} \\ q_{e l}(0)=q_{e l}^{0}\end{array}\right.$

\section{iv. The Two-Body System}

To obtain an interacting system from our two-body non-interacting system, it is necessary to add a boundary condition that prevents the particles from simply going through each other. In this is done by adding a boundary condition on the coincidence set: they set the relative velocity of photon and electron to be 0 whenever the two particles are at the same space and time point ${ }^{[11]}$. In other words:

$$
v_{p h}\left(t_{p h}, s_{p h}, t_{e l}, s_{e l}\right)=v_{e l}\left(t_{p h}, s_{p h}, t_{e l}, s_{e l}\right)
$$

when

$t_{p h}=t_{e l}, s_{p h}=s_{e l}$

v. Derivation of Compton Scattering Formula

To better explain the formula used to calculate predicted momentum of particles in the system, we present a short derivation of the original Compton Scattering Formula in our one-dimensional setting. Assume we have positive energy plane waves going into and coming out of the scattering zone. Assume that conservation of momentum and energy still hold. 
Conservation of momentum means:

$k_{p h}^{i n}+k_{e l}^{i n}=k_{p h}^{o u t}+k_{e l}^{o u t}$

Going into the rest frame of electron, $k_{e l}^{i n}$ becomes 0 . Hence:

$k_{p h}^{\text {in }}=k_{p h}^{\text {out }}+k_{e l}^{\text {out }}$

Suppose the photon is approaching the electron from the right. Thus $k_{p h}^{i n}>0$. Conservation of energy is given by:

$$
\hbar k_{p h}^{i n}+m_{e l}= \pm \hbar k_{p h}^{\text {out }}+\sqrt{m_{\text {el }}^{2}+\hbar^{2}\left(k_{\text {el }}^{\text {out }}\right)^{2}}
$$

(Here and henceforth, we have chosen units in which the speed of light in vacuum is $c=1$.) Substituting EQUATION 2.6 into EQUATION 2.7 and attempting to solve for $k_{p h}^{\text {out }}$ we observe that choosing the plus sign in the right-hand-side of EQUATION 2.7 yields no solution. Thus, the only option is to choose the negative sign, which implies that $k_{p h}^{\text {out }}<0$ since we have assumed that the plane waves have positive energy. As a consequence, the direction of motion of the photon has changed after the scattering event.

Solving for $k_{p h}^{\text {out }}$ then yields the scattering formula:

$k_{p h}^{\text {out }}=-\frac{k_{p h}^{i n}}{1+\frac{2 \hbar}{m_{e l}} k_{p h}^{i n}}$

We note that this agrees with the three-dimensional Compton scattering formula derived in the 1923 paper if the scattering angle in that formula is set to $\pi$. This formula also suggests that the frequency of the scattered $\mathrm{X}$-ray will be lower than the frequency of the incident ray and that the difference in the momentum and energy is transferred to the electron. More precisely, combining EQUATION 2.8 with EQUATION 2.6 and recalling that $\omega=m_{e l} c / \hbar$ one obtains:

$$
k_{e l}^{o u t}-k_{e l}^{i n}=k_{p h}^{i n}-k_{p h}^{o u t}=2 k_{p h}^{i n}\left(\frac{\omega+k_{p h}^{i n}}{\omega+2 k_{p h}^{i n}}\right)
$$

\section{RESULTS}

Throughout the results section, it is important to note that time is measured in attoseconds $\left(10^{-18}\right.$ seconds), and length is measured in units of approximately 3 angstroms $\left(2.997924 \times 10^{-10}\right.$ meters). In these units the speed of light in vacuum is equal to 1 unit of length over time.

\section{i. Single Photon Motion}

The photon probability density looks like this:

HTTPS://REU.DIMACS.RUTGERS.EDU/ AAS377/PHOTON_PDF.MP4

Varying the standard deviation of the initial profile gives us the following:

HTTPS://REU.DIMACS.RUTGERS.EDU/ AAS377/MULTIPLE_PHOTON_PDF.MP4

FIGURE 2 shows multiple trajectories, each with slightly different initial positions.

\section{ii. Single Electron Motion}

The evolution of the probability distribution of the electron's position can be found here:

HTTP://REU.DIMACS.RUTGERS.EDU/ AAS377/ELECTRON NEW.MP4

FIGURE 3 shows the trajectories of many electrons, each in a single-body system guided by its single wave function through its guiding equation, with different initial conditions for each electron. Unlike the trajectories of the photon, the trajectories of the electron exhibit oscillations and travel slower than the speed of light.

\section{iii. Non-Interacting Two-Body System}

Since tensor products preserve probability distributions, "multiplying" the probability density movies of the photon and electron gives the joint probability density for the non-interacting system. Since there is no interaction, we expect the joint probability density to be simply the product of the probability density of a single electron and that of a single photon. The numerical results confirm our expectation.

HTTPS://REU.DIMACS.RUTGERS.EDU/ AAS377/NON_INT_PDF_MESH_NEW.MP4

By solving the system of guiding equations, we obtain FIGURE 4, which shows the trajectories of a non-interacting system of one electron and one photon. 

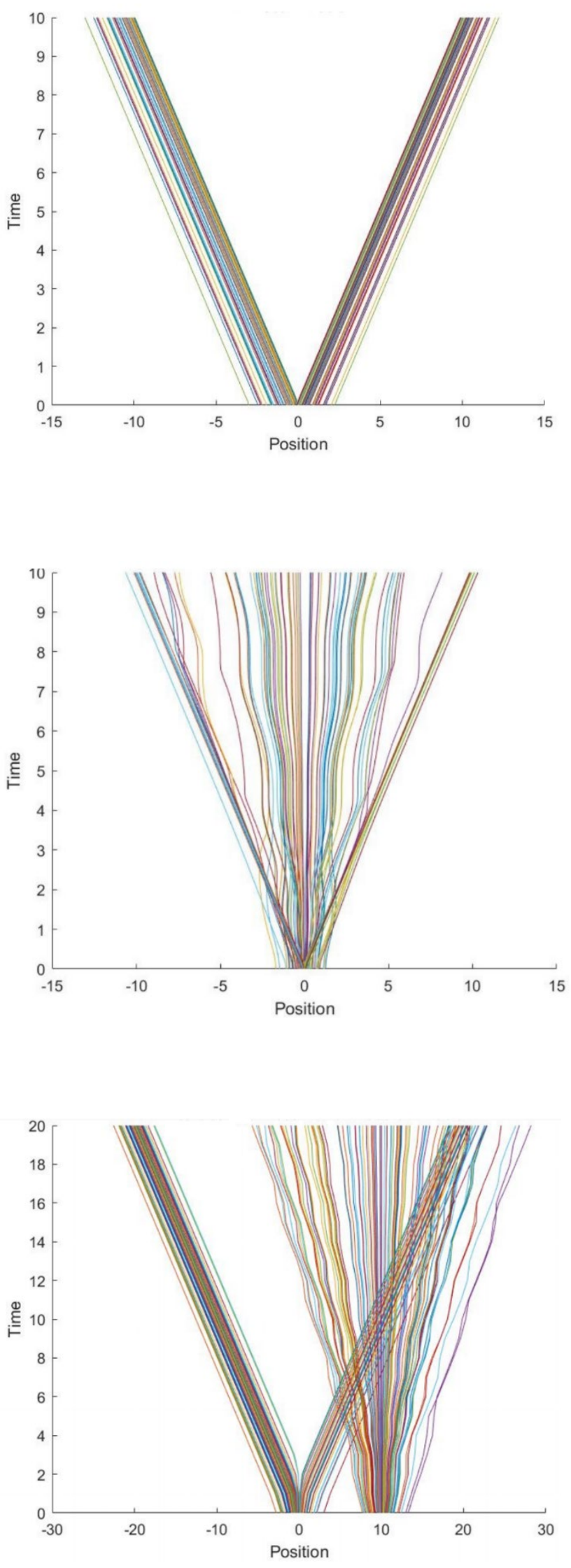

TOP

FIGURE 2: A free photon in one dimension would move either left or right at the speed of light. The linear trajectories in the diagram validates this intuition. Trajectories with different initial conditions are differentiated by color.

MIDDLE

FIgURE 3: A free electron in one dimension moves according to its wave function at a velocity slower than the speed of light. Trajectories with different initial conditions are differentiated by color.

BOTTOM

FIGURE 4: Non-interacting photon (LEFT) and electron (RIGHT) trajectories. Since the two particles do not interact, they pass through one another as the diagram indicates. Trajectories with different initial conditions are differentiated by color.

BELOW

FIGURE 5: Interacting and non-interacting photon (RED) and electron (BLUE) trajectories. The interaction resembles a collision, after which the two particles bounce away from each other. Dotted lines show the non-interacting case, and the solid lines show the interacting case.

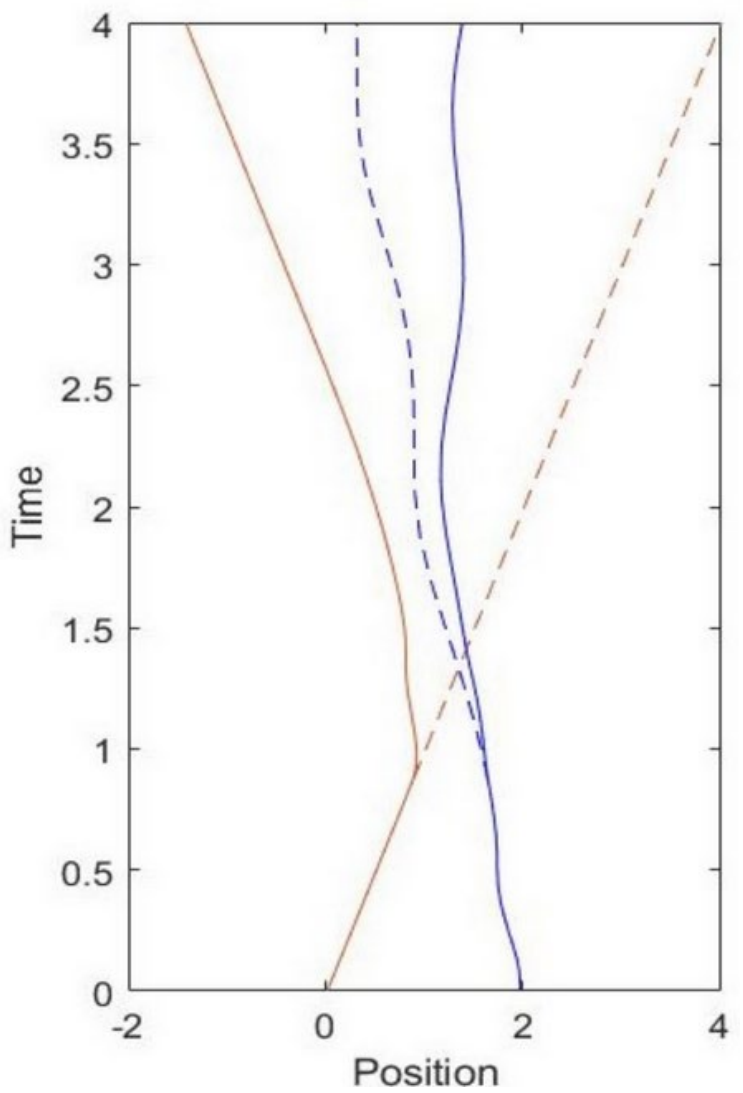



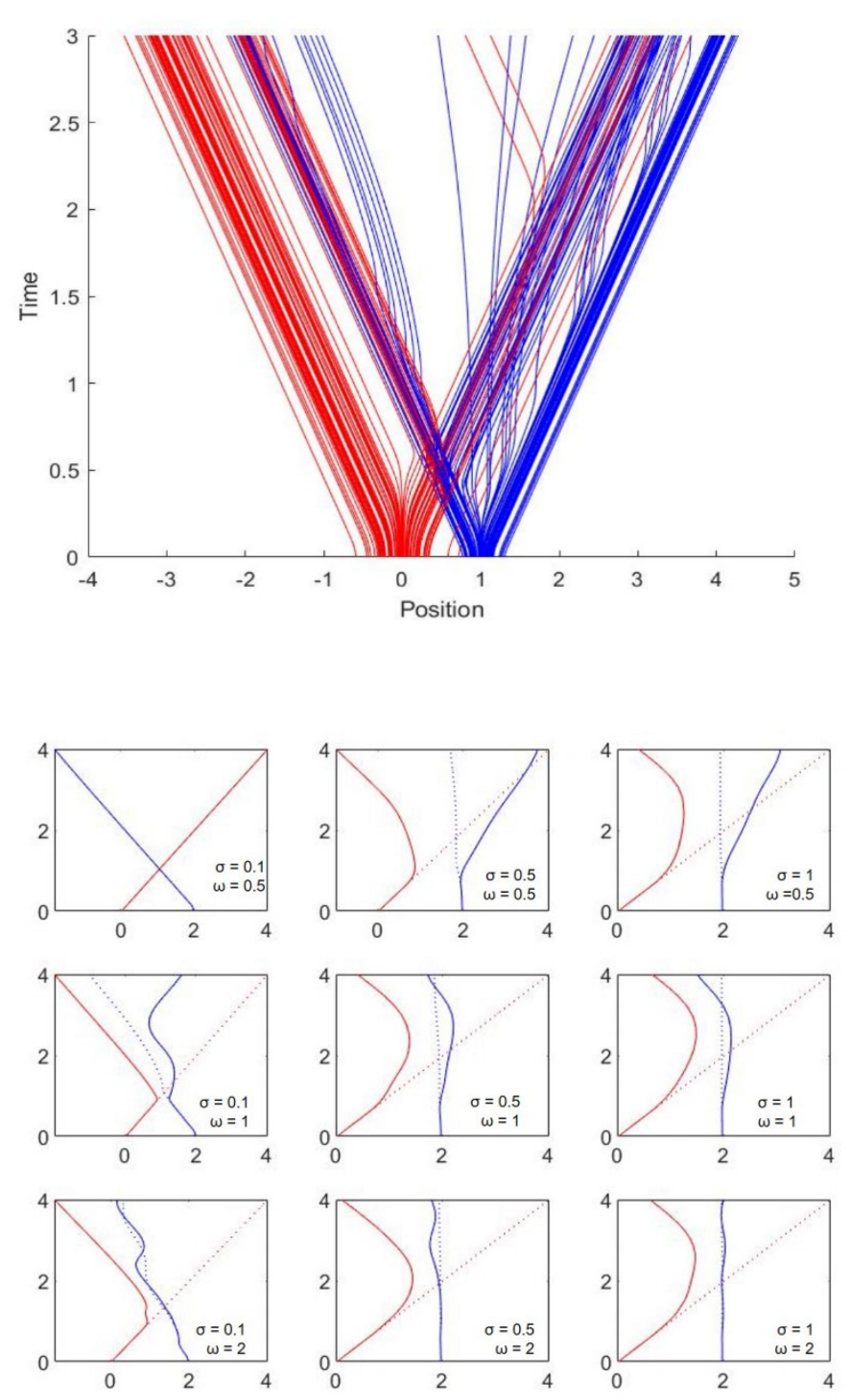

iv. Interacting Two-Body System

Adding the boundary condition to the wave function gives us a modified probability density function:

HTTP://REU.DIMACS.RUTGERS.EDU/ AAS377/INT_PDF_MESH_NEW.MP4

FIGURE 5 is the contour map. The shape of the interacting probability density is delineated by lines from the perspective of someone looking down from above:

HTTP://REU.DIMACS.RUTGERS.EDU/ AAS377/INT_PDF_MESH_NEW.MP4
TOP

FIGURE 6: 100 Overlaid interacting photon (RED) and electron (BLUE) trajectories.

BOTTOM

FIGURE 7: Looking across each row, we can see variation in standard deviation of initial distribution of the electron's position, $\sigma$, while the frequency of the electron, $\omega$, is kept constant across each row. Looking down each column, we can see variation in the frequency of the electron, w!, as the standard deviation of the initial distribution of the electron's position, $\sigma$, is kept constant.

These are the trajectories of the interacting electronphoton system with the boundary condition added in. The solid lines show the interacting system trajectories and the dotted lines show the non-interacting trajectories.

Plotting 100 overlaid trajectories of the interacting system gives FIGURE 6. To make this clearer, here is a video of the collision trajectories being plotted individually on the same set of axes.

HTTP://REU.DIMACS.RUTGERS.EDU/ AAS377/INT_TRAJS_SLOWMO.MP4

\section{v. Varying the Electron Parameters}

Here, we sought to perform numerical experiments using our model. The units used in calculating the values of our parameters are chosen such that Planck's Constant $\hbar=1$ unit of mass $\times$ length squared over time: $\hbar=\frac{\text { Mass } \times \text { Length }}{2}$. In our units, one unit of mass is $0.117337 \times 10^{-32} \mathrm{~kg}^{[a]}$. The parameter $\omega=\frac{m_{e l} c}{\hbar}=\frac{1}{\lambda}$, where $\lambda$ is the reduced Compton Wavelength of the electron and equal to approximately 0.001288 units of length. Note that change in $\omega$ corresponds to a change in mass of electron $m_{e l}$ since the units are set such that $c$ and $\hbar$ are set to equal 1 . Since $\omega$ is the reciprocal of wavelength, we

\footnotetext{
${ }^{[a]}$ For reasons having to do with numerical computations, the value we use for $\omega$ is numerically equal to the mass of the electron but is not the actual physical value. The actual mass of an electron in our units is 776.343694 units of mass, but we are working on the order of 1 unit of mass. That is to say that we are working with an electron mass that is 700 times smaller.
} 
can refer to $\omega$ as electron frequency. Change in $\sigma$ refers to change in the standard deviation of the initial probability distribution of the electron's position in one-dimension, i.e. the standard deviation of the Gaussian distribution representing initial data. The units of $\sigma$ are the same as the units for position: approximately 3 angstroms. The changes in these parameters are visible in FIGURE $7^{[b]}$.

\section{vi. Varying the Phase Angle of Interaction}

In the boundary condition that provides the interaction, it is possible to specify a phase angle. It seems there are no changes as a result of variation in this parameter; however, future research is necessary to elucidate whether this parameter truly has no effect on trajectories. It seems to be that the three graphs in FIGURE 8 are the same since they look like one set of trajectories instead of three when overlaid.

vii. Varying Momentum of Incoming Photon

Changing the momentum of the incoming photon gives us a different picture and lays the groundwork for analyzing Compton Scattering in our setting. The units of momentum are mass $x$ velocity. Our unit of mass is $0.117337 \times 10^{-32} \mathrm{~kg}$, and our unit of velocity is $2.997924 \times 10^{-8} \mathrm{~m} / \mathrm{s}$ (the speed of light in a vacuum). Hence, one unit of momentum is $0.351767 \times 10^{-24} \mathrm{~kg} \mathrm{~m} / \mathrm{s}$.

In order to get a more detailed picture, we varied the mean momentum of the incoming photon between 0 and 10 with mesh of 0.5 . The result is FIGURE 10.

\section{viii. Compton Scattering in Our Model}

We have found some evidence of the applicability of Compton's Scattering Formula to our model, as seen in FIGURE 11. The figure corresponds to the parameter values $\omega=2$ and $\sigma_{p h}=\sigma_{e l}=0.1$, while the initial photon mean frequency is increased from $k_{0}=1$ to $k_{0}=10$, and the resulting electron trajectories are distinguished by color. Again, the unit of momentum is $0.351767 \times 10^{-24} \mathrm{~kg} \mathrm{~m} / \mathrm{s}$.
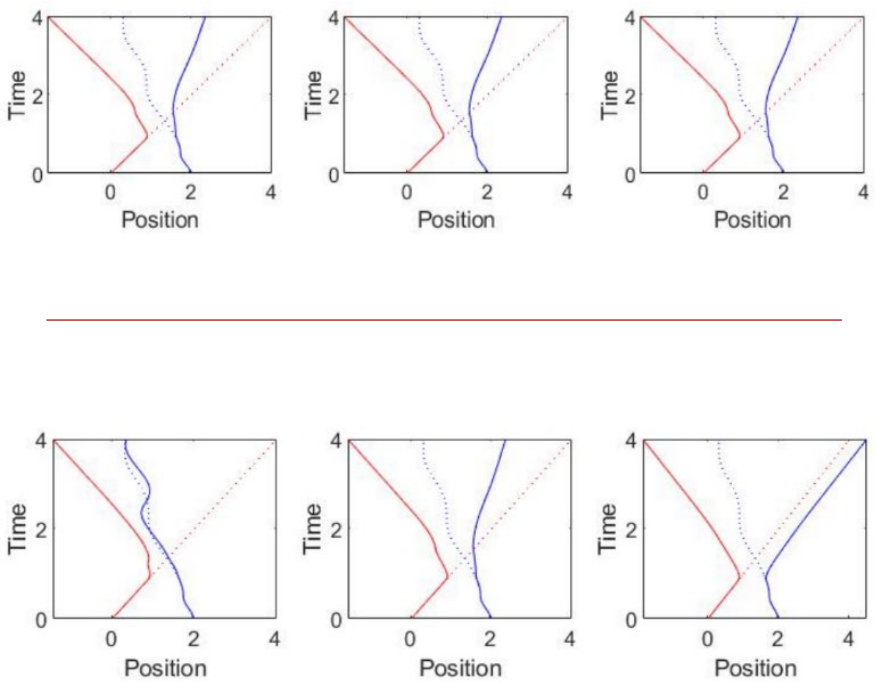

TOP

FIGURE 8: The left-most plot shows an angle of interaction of 0 . The middle plot shows an angle of interaction of $\pi / 2$ and the right-most plot shows an angle of interaction of $\pi / 4$. We can see that as the angle of interaction varies, no change occurs in the particle trajectories. Non-interacting trajectories are visible in dotted lines, and all other parameters are kept constant.

\section{BOTTOM}

FIGURE 9: Interacting photon (RED) electron (BLUE) trajectories with varying photon momentum. In the left-most plot, the momentum of the incoming photon is 0.1 ; in the middle plot, the momentum of the incoming photon is 1, and in the right-most plot, the momentum of the incoming photon is 10. Thus, we see that a higher energy photon-a photon with higher momentum-gets the electron to bounce away. Again, non-interacting trajectories are visible in dotted lines.

\footnotetext{
${ }^{[b]}$ We are of course aware that electron's rest mass is fixed. The point here is to do a mathematical study of how the trajectories would look like for a hypothetical particle with the same characteristics of the electron but a different rest mass.
} 


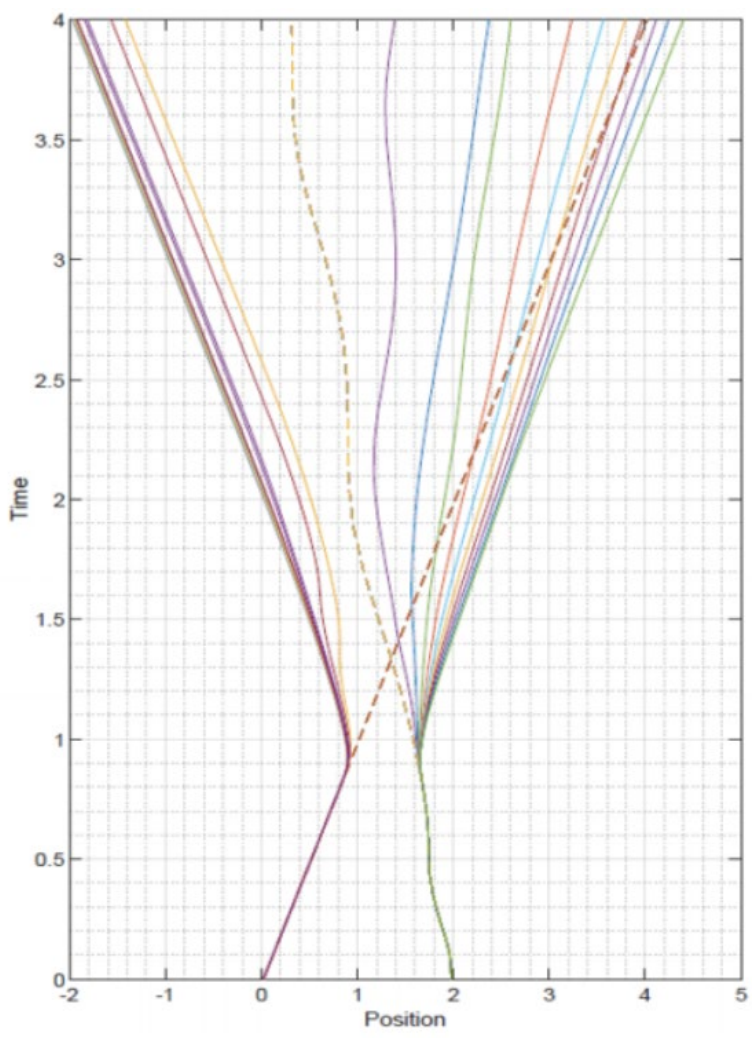

TOP

FIGURE 10: Each pair of trajectories depicts a variation in the momentum of the incoming photon.

BOTTOM

FIGURE 11:

LEFT: Change in Bohmian trajectories of the two particles due to the increase in incoming photon frequency. RIGHT: The corresponding change in electron's post-scattering momentum, computed from those trajectories.
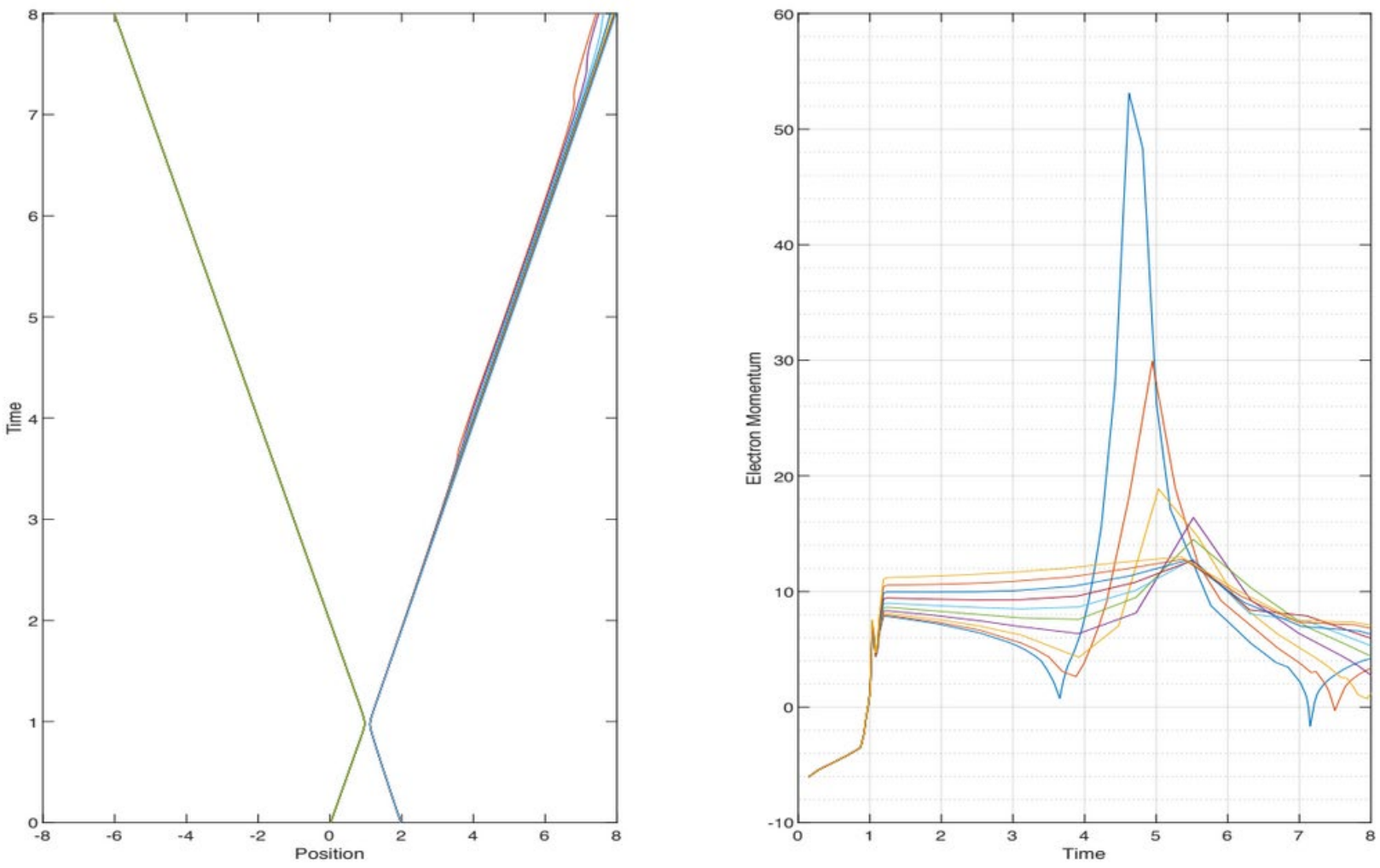

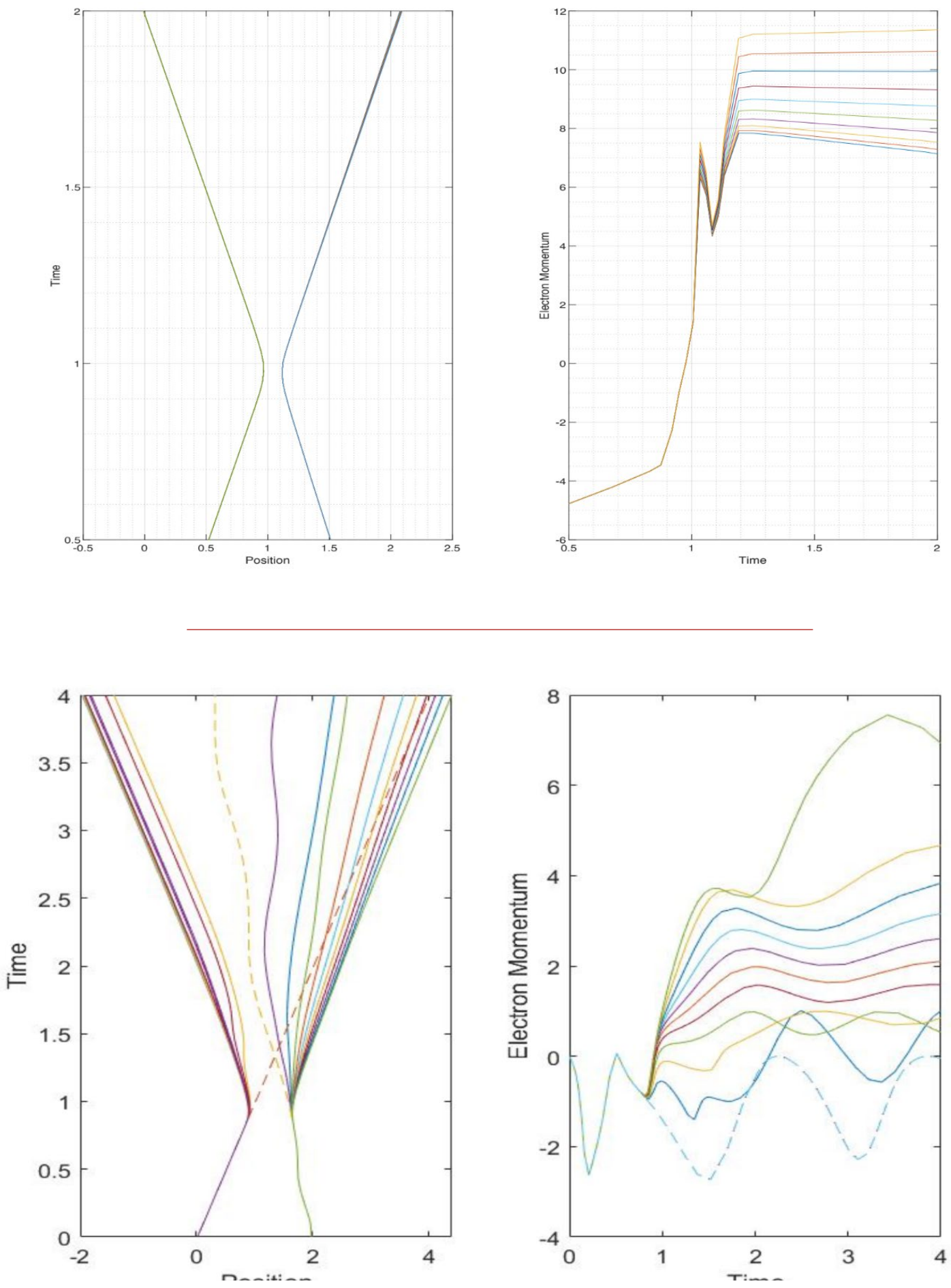

TOP

FIGURE 12: Change in electron momentum due to scattering (detail).

\section{BOTTOM}

\section{FIGURE 13:}

LEFT: Dotted lines show the non-interacting case, and solid lines show interacting cases at various incoming photon frequencies. RIGHT: Solid lines refer to momentum of the outgoing electron at various frequencies of incoming photon. The dotted line shows the momentum of the electron in the non-interacting case, as the two particles "go through" each other. 
If we zoom in on the interaction region, we clearly see that the gain in electron momentum increases linearly with $k_{0}$, in qualitative agreement with Equation 2.9. See Figure 12. In these figures we have calculated the electron's "post-collision" momentum by

$$
P_{e l}=\frac{m_{e l} v_{e l}}{\sqrt{1-\frac{v_{e l}^{2}}{c^{2}}}}
$$

where $v_{e l}$ is the Bohmian velocity of the electron calculated from the wave function, as in EQUATION 2.2, and evaluated at the actual positions of the two particles that have been calculated by solving the two differential equations Equation 2.1 and EQuation 2.2. The graph on the right of FIGURE 13 shows that even in the non-interacting case, the momentum of the electron computed from trajectories is not constant because its Bohmian velocity is not constant; instead, it fluctuates in time.

Finally, we compared our computed electron momentum to the theoretical values computed with Compton's Formula. Our results show that some frequencies of the incoming photon allow for a better match than others between Compton's Formula and computations based on De Broglie-Bohm theory. Further research in this area would be useful to understand which regime of parameters allow for a better fit, and why.

\section{DISCUSSION}

In this research, we studied probability density functions and Bohmian trajectories for a single electron and a single photon guided by their respective wave functions in one space dimension ${ }^{[10,12]}$. We then analyzed the probability density functions and Bohmian trajectories of both a non-interacting and interacting two-body system consisting of a photon and an electron, in one space dimension, where both particles were guided by the same two-body multi-time wave function.

Dirac introduced the concept of multi-time wave functions, but the idea that the wave function guided the motion of particles in the system did not arise until Bohmian Mechanics was developed ${ }^{[2,7]}$. The idea that multi-time wave functions can guide the motion of relativistic particles is far more recent ${ }^{[8]}$.

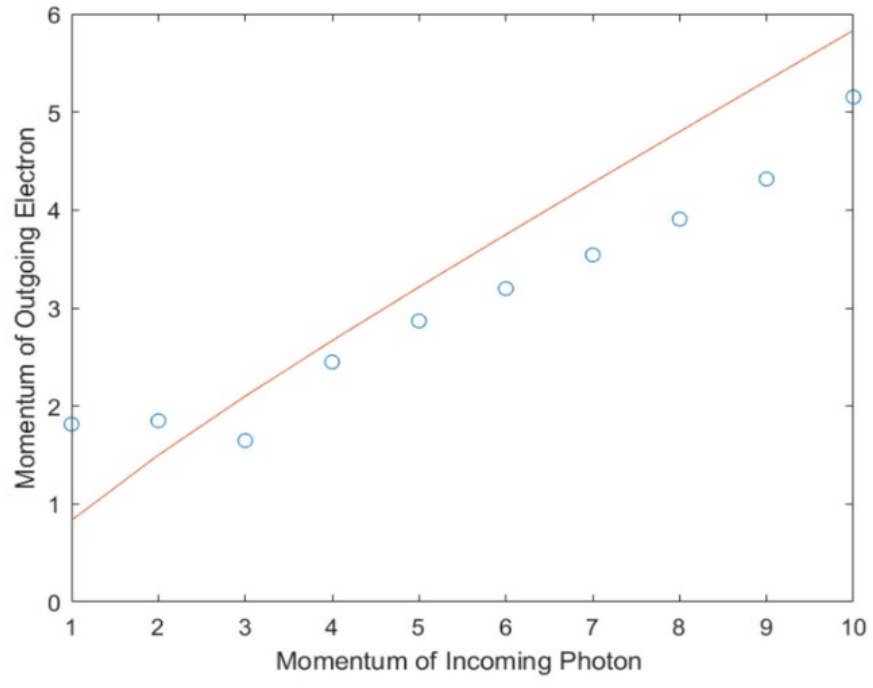

FIGURE 14: The red line shows theoretical values of momentum of the outgoing electron computed using Compton's Formula. The blue circles indicate the results of our numerical investigation of the outgoing electron momentum computed from Bohmian trajectories.

We studied how the dynamics of the two particles depended on several key parameters in the problem, such as the frequency of the incoming photon, and the rest mass of the electron. We did a preliminary analysis of Compton scattering, and found qualitative agreement between Compton's quasi-classically derived formula for the gain in electron's momentum, and the corresponding values computed from the trajectories. However, there is much work that remains to be done.

In our modeling of the two-body system, the trajectories of the non-interacting system were shown to simply be a superposition of the trajectories of a single photon and a single electron. This was no longer the case once the boundary condition was added. The wave function governing the system indicates that post-interaction, the two particles become entangled even if we initially have a pure product state.

In varying the parameters of mass and standard deviation of the initial distribution for the electron, we saw that more massive electrons were likely to have a straighter trajectory, indicating slower 
movement and less likelihood to recoil away. Furthermore, varying the standard deviation of the initial distribution of the electron's position seemed to alter the trajectories and the nature of the interaction: lower standard deviation values resulted in the electron and photon staying together for longer instead of bouncing off immediately. However, more investigation is required to better understand the interplay between these parameters. In varying the phase angle of interaction, we found evidence that the dynamics of the interaction is independent of that phase angle. Our work in varying the momentum of the incoming photon shows that shooting a higher energy photon at the electron will proportionally increase the electron's post-collision momentum in a manner consistent with Compton's formula.

\section{i. Analysis of Compton Scattering Results}

It should be noted that we cannot expect the actual electron momenta to be uniformly close to the values predicted by EQUATION 2.9, since this formula is of a classical nature (apart from using Einstein's suggested values for the momentum and energy of the photon). It has been derived using the rules of classical mechanics, such as exact conservation of a welldefined energy and momentum for both particles. For instance, in the example above, the initial electron distribution has a standard deviation of 0.1 ; therefore, by the Uncertainty Principle, the standard deviation of its momentum distribution will be at least $5 \sim$. If we randomize the initial position of the electron according to the initial distribution, as we are supposed to do in a quantum theory, we cannot expect the electron momentum at any later time to be close to any one particular value. It is only expected to fluctuate around some mean. This would be equally true for a non-interacting electron. It is a hallmark of the quantum nature of elementary particles.

We would nevertheless like to be able to derive a quantum-mechanical (i.e. appropriately statistical) version of formula EQUATION 2.9 within our framework. There are, however, at least two major obstacles on the way to defining a notion of momentum for a quantum particle, particularly for a photon.
Firstly, our theory does not admit plane waves since these are not square-integrable, and therefore do not belong to our Hilbert space. Instead, we work with Gaussian wave packets. Secondly, after interaction, the system is entangled. We may start with a wave function that is a pure product, but we no longer have a pure product after the photon and electron interact. Thus, even if we could start with plane waves going in, we would not have plane waves coming out, which violates the underlying assumption used to derive EQUATION 2.9. Developing a workable notion of momentum for the particles in our system will be part of our future work.

\section{ii. Future Research}

We plan to investigate ways of defining a notion of momentum in our framework: one that would allow us to derive the one-dimensional analog of the Compton scattering formula in some appropriate classical or semi-classical limit. Defining momentum is easy for a single particle using Fourier transform. There are, however, many difficulties in isolating photonic and electronic properties from the twobody wave function of the system. A potential way to address this might be to use techniques from Linear Algebra to decide whether the wave function of the system becomes locally approximately a pure product far away from the interaction zone.

Naturally, we also plan to expand our analysis to three space dimensions. We hope to eventually arrive at an $\mathrm{N}$-body, relativistic, and quantum mechanical system in three space dimensions from which Maxwell's equations can be derived in the limit as the number of photons goes to infinity

\section{ACKNOWLEDGEMENTS}

We would like to thank the Rutgers University DIMACS REU program and the Rutgers University Math Department for their generous support. We are grateful to Dr. Shadi Tahvildar-Zadeh for his patient guidance, and Parker Hund for our valuable discussions. We thank Erika Melder for her technical support. 


\section{REFERENCES}

[1] Bialynicki-Birula, I. (1996). The photon wave function. In Coherence and quantum optics, VII , 313-322.

[2] Bohm, D. (1952). A suggested interpretation of the quantum theory in terms of hidden variables. part i. Phys. Rev., 85 , 166-179.

[3] Compton, A. (1923). A quantum theory of the scattering of X-rays by light elements. Physical review , 21(5), 483.

[4] de Broglie, L. (1928). La nouvelle dynamique des quanta. In J. Bordet (Ed.), Cinquième conseil de physique Solvay. Gauthier-Villars, Paris.

[5] Dirac, P. (1927). The quantum theory of the emission and absorption of radiation. Proc. Roy. Soc. Lond., 14(767), 243265.

[6] Dirac, P. (1928). The quantum theory of the electron. Proc. Roy. Soc. Lond., A 117, 610624.

[7] Dirac, P. (1932). Relativistic quantum mechanics. Proc. Roy. Soc. Lond., 136(829), 453-464.

[8] Dürr, D., Goldstein, S., Munch-Berndl, K., \& Zanghí, N. (1999). Hypersurface Bohm-Dirac models. Phys. Rev. A, 60, 2729-2736.

[9] Dürr, D., Goldstein, S., \& Zanghí, N. (2013). Quantum physics without quantum philosophy. New York: Springer.

[10] Dürr, D., \& Teufel, S. (2009). Bohmian mechanics: The physics and mathematics of quantum theory. New York: Springer.

[11] Kiessling, M. K. H., Lienert, M., \& Tahvildar-Zadeh, A. S. (2019). A Lorentz-covariant interacting electron-photon system in one space dimension. preprint [arXiv: 1906.03632].

[12] Kiessling, M. K. H., \& Tahvildar-Zadeh, A. S. (2018). On the quantum mechanics of a single photon. J. Math. Phys., 59 (112302).

[13] MATLAB. (2010). version 7.10.0 (r2010a). Natick, Massachusetts: The MathWorks Inc.

[14] Penrose, R. (2016). Fashion, faith, and fantasy in the new physics of theuniverse. Princeton University Press.

[15] Weinberg, S. (1995). The quantum theory of fields, vol. I. Cambridge Univ. Press. 